\title{
THE PHTHALEIN TEST IN ORTHOSTATIC ALBUMINURIA *
}

\author{
T. C. HEMPELMANN, M.D. \\ ST. LOUIS
}

Orthostatic albuminuria is a comparatively common condition, especially in children, and has been the subject of frequent investigation, yet the pathology of the condition is far from being clearly understood. Although it is not commonly classified among the nephritides, actual opportunities for histologic examination of kidneys from individuals with orthostatic albuminuria, have been very infrequent, chiefly because of the benign character of the affection. Such opportunities for necropsy examination as have occurred have failed to reveal any of the usual pathologic findings of a nephritis. Until the accumulation of necropsy findings or physiologic experimentation shall have cleared up the pathogenesis of the condition, we must rely chiefly on clinical observation and renal tests for our knowledge of the factors involved in its production.

In a clinical investigation, the question of the integrity of the kidney function is naturally one of the first to suggest itself. A few earlier studies of kidney function in orthostatic albuminuria, using salt, potassium iodid, or indigo-carmin excretion, etc., as tests, have been made, but the results were indefinite and inconclusive. So far as I know, no tests of renal function have been made with the more delicate methods introduced in recent years, and hence this study was undertaken. In a preliminary note ${ }^{1} \mathrm{I}$ reported briefly the results of the phenolsulphonephthalein test in four cases, which are included in this paper.

Only marked cases of the so-called lordotic type were used in these studies-cases in which large amounts of albumin appeared in the urine after such a simple procedure as having the child stand against the wall for ten minutes with the heels and back of the head touching the wall, thus throwing the shoulders well back, and bringing the lumbar spine forward. In all these cases the urine was free from a'bumin after a night's rest in bed. Children with slight or moderate degrees of orthostatic albuminuria were not used for tests. In all

* Submitted for publication Sept. 18, 1915.

* From the Department of Pediatrics, Washington University, and the St. Louis Children's Hospital.

1. Read before the Washington University Medical Society, May 11, 1914; reported in the Journal of the Missouri Med. Soc., June, 1914, and the Bulletin of the Washington University Med. School, June, 1915. 
cases the albumin precipitated on the addition of acetic acid, although this precipitate was usually increased by heating. No casts were found in the arine.

The manner of making the tests was as follows: The cases were referred from among the children coming to the out-patient dispensary and admitted to the hospital for the carrying out of the tests. On admission, the children were kept in bed over night and the morning urine examined for albumin. If free, the child was placed in the position described above for ten or fifteen minutes, and the urine examined again. Cases then showing large amounts of albumin (enough to give a curdy precipitate with the acetic acid test), were utilized. The patient was again put to bed and the following day-the child meanwhile not being allowed up-a phenolsulphonephthalein test was made with the child in the position of accentuated lordosis throughout the whole time of the test. In the test $0.6 \mathrm{gm}$. of phenolsulphonephthalein contained in 1 c.c. of solution, was injected into the muscles of the back, as advised by Rowntree and Geraghty. The urine excreted in the first and second hour after injection was collected separately and the amount of phenolsulphonephthalein present in each estimated by means of a Du Boscq colorimeter. The children were then allowed to play about the ward for two or three days, an interval of time sufficient to get rid of the phthalein used in the first injection. On the third or fourth day, after the child had been in bed over night, the test was repeated with the child in the normal position, that is, lying flat in bed, not accentuating the lumbar curve. In some of the cases, the time of first appearance of the drug in the urine was noted, but not in all. The position of accentuated lordosis was maintained during the two hours required for the test by having the child lie on its back with several hard pillows under the lumbar spine, or by sitting in a chair, with a stick across the back, held in the crooks of the elbows. In three instances the order of the tests was reversed, the test in the normal position being made first, and in one case a longer interval elapsed between tests. The protocols are as follows:

\section{PROTOCOLS}

CASE 1.-Richard P. (No. 3828), aged 12 years, came to the dispensary in January, 1913, because of enuresis. He presented the typical findings of an orthostatic albuminuria, all other physical examination being negative except for an accidental heart murmur, which disappeared during the period of observation. The first phthalein test was made Feb. 19, 1913, with the patient in bed. $^{2}$ The first appearance of the drug was in five minutes, 62.8 per cent. being excreted in the first hour, and 5 per cent. in the second, or 67.8 per cent.

2. It will be remembered that the drug normally appears in the urine in from five to eleven minutes after intramuscular injection, 40 to 65 per cent. being excreted in the first hour after the initial appearance, and 65 to 80 per cent. in two hours. 
in two hours. The test Feb. 22, 1913, with the patient in the position of accentuated lordosis gave the first appearance in eight minutes, with 42.8 per cent. excreted in the first hour and 10 per cent. in the second, or 52.8 per cent. in the two hours; that is, 20 per cent. less in the first hour and 15 per cent. less in the two hours, than when he was in the normal position. April 11, 1914, the test was repeated, with the patient in the normal position, when 59 per cent. was excreted in the first hour and 5 per cent. in the second, or 64 per cent. in the two hours - a normal output, and no evidences of nephritis a year after the first tests.

CASE 2.-Blanche C. (No. 3302), 11 years old, came to the dispensary because of enuresis. Typical orthostatic albuminuria with no other abnormality. First phthalein test on May 4, 1914, with the patient in the position of accentuated lordosis. Time of appearance not noted. First hour, 39 per cent. excreted, second hour, 20 per cent., or 59 per cent. in two hours. Second test on May 5, 1914, with patient in the normal position. First hour, 48.8 per cent. excreted; second hour 10 per cent., or 58.8 per cent. in the two hours; that is, in the lordotic position 10.8 per cent. less in the first hour, but an equivalent amount in the two hours.

CASE 3.-Robert M. (No. 5027), 9 years old, came to the dispensary Feb. 18, 1914, because of enuresis and trachoma. Physical examination reveals no other abnormalities except an orthostatic albuminuria. Phthalein test March 11, 1914, in position of accentuated lordosis, showed first appearance in eight minutes, with 35 per cent. excreted in the first hour and 5 per cent. in the second, or 40 per cent. in the two hours. Phthalein test May 6, 1914, normal position, showed $66 \% 3$ per cent. excreted in the first hour, 20 per cent. in the second, or $86 \%$ per cent. in the two hours; that is, in the lordotic position, over 30 per cent. less for the first hour and 46 per cent. less for the two hours.

CASE 4.-Theodore K. (No. 3216), 10 years, came to the dispensary in May, 1910, because of hypertrophied tonsils and adenoids, with enuresis. He had, and still has, a typical orthostatic albuminuria, which has been observed now for over five years, with no further evidences of a nephritis. Subjected to the phthalein test May 2, 1914, in the position of accentuated lordosis. First hour, 41.2 per cent. excreted and in the second hour 10 per cent., or 51.2 per cent. for the two hours. Test repeated May 6, 1914, with boy in normal position, when 48.3 per cent. was excreted in the first hour, and 15 per cent. in the second, or 63.3 per cent. in the two hours. This case, therefore, shows approximately 12 per cent. less excreted for the two hours, and 7 per cent. less the first hour while in the lordotic position.

CASE 5.-John M. (No. 8192), aged 10 years, came to the dispensary because of urticaria and adenoids. No other abnormality except an orthostatic albuminuria. First phthalein test March 26, 1915, with boy in normal position. Time of appearance eight minutes. First hour, 55 per cent. excreted; second hour, 15 per cent. excreted, or 70 per cent. in two hours. Second phthalein test March 31, 1915, lordotic position. Time of appearance, eleven minutes. First hour, 30 per cent. excreted; second hour 40 per cent. excreted, or 70 per cent. in the two hours. In the lordotic position, 25 per cent. less was excreted during the first hour, but an equal amount for the two hours.

CASE 6.-Francis M. (No. 8317), aged 8 years, came to the dispensary because of an aphthous stomatitis. Typical orthostatic albuminuria in addition. First phthalein test April 16, 1915, in the lordotic position. Time of appearance, eight minutes. First hour 45 per cent. excreted, and second hour 15 per cent. excreted, or 60 per cent. in the two hours. Second phthalein test April 19, 1915, with boy in the normal position. First appearance, six minutes. First hour 60 per cent. excreted, second hour 10 per cent., or 70 per cent. excreted in the two hours. There was, therefore, 15 per cent. less excreted in the first hour and 10 per cent. less in two hours while in the lordotic position. 
CASE 7.-Rosie T. (No. 8406), aged 11 years, came to the dispensary for bronchitis and orthostatic albuminuria. First phthalein test May 1, 1915, in normal position, showed 55 per cent. of the drug excreted in the first hour, and 12 per cent. in the second, or 67 per cent. in two hours. Second test on May 3, 1915 , in the lordotic position, with 40 per cent. of the drug excreted in the first hour and 20 per cent. in the second, or 60 per cent. in the two hours. There was, therefore, 15 per cent. less excreted during the first hour in the lordotic position, and 7 per cent. less in the two hours.

Control 1.-Frank R. (No. 3910), normal, well boy, subjected to the phthalein test Feb. 19, 1913, in position of accentuated lordosis and showed 56.5 per cent. excreted in the first hour and 15 per cent. in the second, or 71.5 per cent. in the two hours. Test repeated Feb. 22, 1913, with patient in the normal position, when 55 per cent. was excreted in the first hour and 15 per cent. in the second, or 70 per cent. in the two hours - approximately the same result in both positions.

ControL 2.-Alvin J. (No. 5572), aged 7 years, up and about the wards, with no urinary abnormality whatever. Subjected to the phthalein test in the position of accentuated lordosis May 6, 1914, and showed 76.8 per cent. of the drug excreted in the first seventy minutes (was unable to void at sixty minutes) and 5 per cent. in the next fifty minutes, or 81.8 per cent. in two hours. Test repeated with the boy in the normal position May 8, 19.14, when 71.3 per cent. was excreted in the first hour and 12.5 per cent. in the second, or 83.8 per cent. in the two hours; that is, approximately the same for the two-hour intervals, but 5.5 per cent. more in seventy minutes, lordotic position than in one hour, normal position.

The accompanying table summarizes the results of the seven cases and controls.

It will first be noticed that all of the cases except Case 2, came within the limits of the normal as regards phthalein output when in the normal position. Case 2 is slightly under the average, but hardly enough to call the phthalein output abnormal. The seven cases averaged 69 per cent. output in two hours and 56.6 per cent. in the first hour. It was found, however, that when the child with orthostatic albuminuria maintained the lordotic position during the test, there was apparently a slowing up of the excretion of phthalein. This was most marked in the first hour, when the average for the seven cases was 39 per cent., in contrast to 56.6 per cent. in the normal position-a decrease averaging 17.6 per cent. The total difference for the two hours is less, averaging but 12.9 per cent. Moreover, it will be noted that in every instance this retardation occurs in the first hour, the extremes being 7.1 per cent. and 31.6 per cent., but that when the total for the two hours is contrasted, the retardation is less, and in two cases practically the same amount was excreted. It will be noted that in Case 3, the figures for the retardation are extreme, and much higher than the average. If this case is eliminated, the slowing taking place in the first hour and the increased excretion in the second hour, which brings the totals nearer for the two positions, is more 


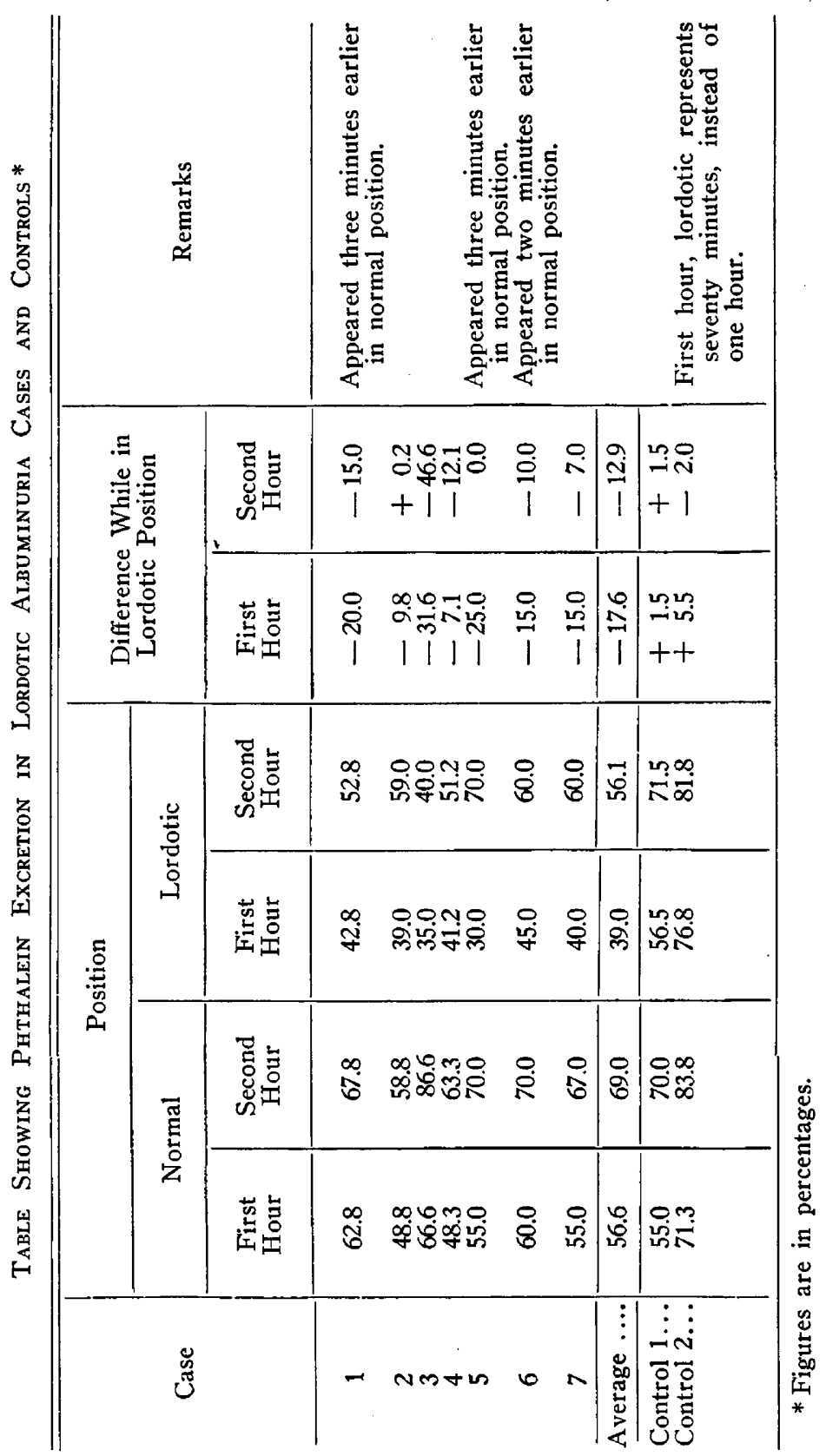


striking. Thus, eliminating this case, an average of 15.6 per cent. less excretion takes place in the first hour, and a total decrease of 7.3 per cent. in two hours. A simple way of stating the finding is that cases of orthostatic albuminuria in bed excreted 56.6 per cent. of phthalein in the first hour and 12.4 per cent. in the second. In accentuated lordosis, a 39 per cent. output took place in the first hour and 17.1 per cent. in the second.

The normal control cases showed no change in elimination between the normal and lordotic positions.

If the above findings may be regarded as lending support to any cf the various theories of pathogenesis, it would probably be that one which assumes a mechanical interference with renal circulation as the chief causative factor in the production of an orthostatic albuminuria. Jehle, who has been the most ardent advocate of this theory, believes that the accentuation of the normal forward curve of the lumbar spine so commonly seen in individuals with orthostatic albuminuria, produces an interference with the renal circulation either by compressing or stretching the renal vessels, or otherwise causing a congestion of the kidneys. He does not deny that vasomotor influences may affect the degree of albuminuria, but believes the mechanical factor to be the main feature, without which there is no orthostatic albuminuria. In support of his contention, he submits evidence to show that effacing the lordosis by postural methods causes a disappearance of the albuminuria, and that, furthermore, it is possible to produce an albuminuria in normal individuals by pressure directed through the abdominal walls against the great vessels, or, in animals, by directly constricting or compressing the renal vessels for a short time. Such methods would produce a retardation of the renal circulation, and would probably delay the excretion of a drug such as phenolsulphonephthalein.

\section{SUMMARY}

Renal function, as measured by the phenolsulphonephthalein test, in children with marked degrees of orthostatic albuminuria, is normal when the patients are at rest in bed. When these patients are placed in a position of accentuated lordosis, producing a marked albuminuria, the total output of phthalein in two hours is reduced-in the above seven cases, on an average 12.9 per cent. The most marked feature, however, is the retardation which takes place in the output during the first hour-the average of our cases being 17.6 per cent. less in the lordotic position. Normal children do not show this retardation and decreased elimination with the change of posture. If this retardation may be brought into relation with any of the theoretical ideas of the 
pathogenesis of orthostatic albuminuria, it would probably be that which associates the condition with a decreased vascular supply to the kidney as the result of posture.

Metropolitan Building.

\section{REFERENCES}

Jehle, L.: Ergebn. d. inn. Med. u. Kinderh., 1913, xii, 808.

Fischl, R., and Popper, E.: Jahrb. f. Kinderh., 1915, 1xxxi, 31.

Langstein, L.: Pfaundler and Schlossmann's Handb. f. Kinderh., Ed. 2, iv, 30 . 